\title{
Clusters of Galaxies: Setting the Stage
}

\author{
A. Diaferio $\cdot$ S. Schindler $\cdot$ K. Dolag
}

Received: 21 September 2007 / Accepted: 28 September 2007 / Published online: 15 February 2008 (C) Springer Science+Business Media B.V. 2008

\begin{abstract}
Clusters of galaxies are self-gravitating systems of mass $\sim 10^{14}-10^{15} h^{-1} \mathrm{M}_{\odot}$ and size $\sim 1-3 h^{-1} \mathrm{Mpc}$. Their mass budget consists of dark matter $(\sim 80 \%$, on average $)$, hot diffuse intracluster plasma $(\lesssim 20 \%)$ and a small fraction of stars, dust, and cold gas, mostly locked in galaxies. In most clusters, scaling relations between their properties, like mass, galaxy velocity dispersion, X-ray luminosity and temperature, testify that the cluster components are in approximate dynamical equilibrium within the cluster gravitational potential well. However, spatially inhomogeneous thermal and non-thermal emission of the intracluster medium (ICM), observed in some clusters in the X-ray and radio bands, and the kinematic and morphological segregation of galaxies are a signature of non-gravitational processes, ongoing cluster merging and interactions. Both the fraction of clusters with these features, and the correlation between the dynamical and morphological properties of irregular clusters and the surrounding large-scale structure increase with redshift.

In the current bottom-up scenario for the formation of cosmic structure, where tiny fluctuations of the otherwise homogeneous primordial density field are amplified by gravity, clusters are the most massive nodes of the filamentary large-scale structure of the cosmic web and form by anisotropic and episodic accretion of mass, in agreement with most of the observational evidence. In this model of the universe dominated by cold dark matter, at the present time most baryons are expected to be in a diffuse component rather than in stars and
\end{abstract}

\footnotetext{
A. Diaferio $(\bowtie)$

Dipartimento di Fisica Generale "Amedeo Avogadro”, Università degli Studi di Torino, Via P. Giuria 1, 10125, Torino, Italy

e-mail: diaferio@ph.unito.it
}

\section{A. Diaferio}

Istituto Nazionale di Fisica Nucleare (INFN), Sezione di Torino, Via P. Giuria 1, 10125, Torino, Italy

\section{S. Schindler}

Institut für Astro- und Teilchenphysik der Leopold-Franzens Universität Innsbruck,

Technikerstrasse 25/8, 6020, Innsbruck, Austria

K. Dolag

Max-Planck-Institut für Astrophysik, Karl-Schwarzschildstr. 1, P.O. Box 1317, 85741,

Garching b. München, Germany 
galaxies; moreover, $\sim 50 \%$ of this diffuse component has temperature $\sim 0.01-1 \mathrm{keV}$ and permeates the filamentary distribution of the dark matter. The temperature of this Warm-Hot Intergalactic Medium (WHIM) increases with the local density and its search in the outer regions of clusters and lower density regions has been the quest of much recent observational effort.

Over the last thirty years, an impressive coherent picture of the formation and evolution of cosmic structures has emerged from the intense interplay between observations, theory and numerical experiments. Future efforts will continue to test whether this picture keeps being valid, needs corrections or suffers dramatic failures in its predictive power.

Keywords Galaxies: clusters: general · Intergalactic medium · Cosmology: large-scale structure of Universe

\section{Preamble}

The present chapter provides the general framework of the physics of clusters of galaxies: it outlines how clusters connect to several astrophysical issues, from cosmology to the formation of galaxies and stars. Some of these topics are discussed in the chapters of this volume; we refer to these chapters when appropriate. When the topic we mention here is not dealt with elsewhere in this volume, we refer to some of the most recent reviews.

\section{The Observational Framework}

\subsection{Cluster Components}

\subsubsection{Mass Content}

The discovery of clusters as concentrations of galaxies on the sky dates back to 1784 , when Charles Messier mentions the Virgo cluster in his Connaissance des Temps (see the fascinating historical review by Biviano 2000). The first systematic optical surveys of clusters appeared almost two centuries later (Abell 1958; Zwicky et al. 1961-1968).

In 1930s, Zwicky, by assuming that the cluster in the Coma constellation, containing hundreds of bright galaxies (Fig. 1) ${ }^{1}$ is in virial equilibrium, found that the mass required to bind the system gravitationally should be roughly hundred times larger than the sum of the masses of the individual galaxies (Zwicky 1933, 1937). In fact, if the cluster is an isolated, spherically symmetric system in dynamical equilibrium, the virial theorem yields the total mass

$$
M=\frac{3 \sigma^{2} R}{G}
$$

where $G$ is the gravitational constant, $\sigma$ is the dispersion of the galaxy velocities along the line of sight, and $R$ is the cluster size

$$
R=\frac{\pi}{2} \frac{N(N-1)}{2}\left(\sum_{i} \sum_{i>j} \frac{1}{r_{i j}}\right)^{-1},
$$

\footnotetext{
${ }^{1}$ See Biviano (1998) for a historical perspective on the Coma cluster.
} 

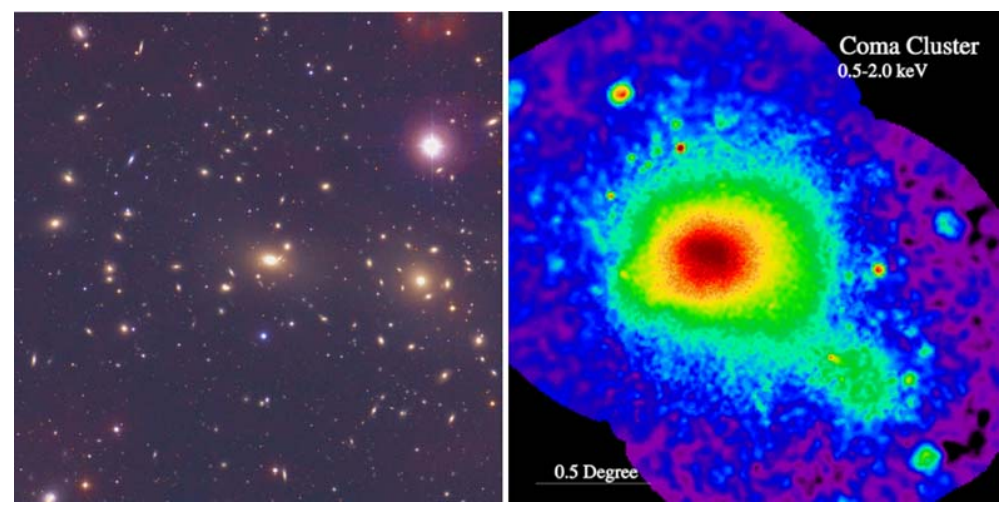

Fig. 1 Left panel: Optical image of the central part of the Coma cluster $(23 \times 23$ arcmin $)$ centered on the galaxy NGC4889; the bright galaxy on the right is NGC4874. Right panel: X-ray image (ROSAT) of Coma $(164 \times 150$ arcmin) (from http://chandra.harvard.edu/photo/2002/0150/more.html)

where $r_{i j}$ is the projected separation between galaxies $i$ and $j$, and $N$ is the number of galaxies; (2) assumes that all the galaxies have the same mass. Clusters are not isolated systems and the correct application of the virial theorem requires the inclusion of a surface term (The and White 1986; Girardi et al. 1998). However, correcting for this factor, and correcting also for departures from spherical symmetry, for galaxies of different masses and for the presence of interlopers, namely galaxies which are not cluster members but appear projected onto the cluster field of view, is not sufficient to fill in the discrepancy between the virial mass and the sum of the galaxy masses. We can reach the same conclusion with a more sophisticated dynamical analysis based on the Jeans equations (Biviano 2006). Zwicky's result was the first indication of the existence of dark matter.

With the advent of astrophysics from space in 1960s, the Uhuru satellite revealed that clusters are the most luminous extended X-ray sources on the sky (Gursky et al. 1972, Fig. 1). The origin of the X-ray luminosity $L_{X}$ was very early interpreted as thermal Bremsstrahlung emission from a hot intracluster plasma (Felten et al. 1966; Bykov et al. 2008b-Chap. 8, and Kaastra et al. 2008-Chap. 9, this issue):

$$
L_{X}=\int n_{\mathrm{e}}(\mathbf{r}) n_{\text {ions }}(\mathbf{r}) \Lambda[T(\mathbf{r})] \mathrm{d}^{3} \mathbf{r},
$$

where $n_{\mathrm{e}}$ and $n_{\text {ions }}$ are the electron and ion number densities in the intracluster medium (ICM) and $\Lambda(T)$ is a cooling function. For temperatures $k T \gtrsim 2 \mathrm{keV}$ ( $k$ is the Boltzmann constant), when the ICM is almost fully ionised, we have $\Lambda(T) \propto T^{1 / 2}$. The existence of a hot diffuse X-ray emitting gas implies the presence of a deep gravitational potential well that keeps the gas confined. This evidence made untenable the hypothesis that clusters are chance superpositions of galaxies or expanding systems (Ambartsumian 1961). By assuming hydrostatic equilibrium and spherical symmetry, the cumulative mass within radius $r$ is

$$
M(<r)=-\frac{k T r}{G \mu m_{\mathrm{p}}}\left(\frac{\mathrm{d} \ln \rho_{\mathrm{gas}}}{\mathrm{d} \ln r}+\frac{\mathrm{d} \ln T}{\mathrm{~d} \ln r}\right),
$$

where $\mu$ is the mean molecular weight, $m_{\mathrm{p}}$ the proton mass and $\rho_{\text {gas }}$ the gas mass density. This type of analysis confirmed that at least $70 \%$ of the cluster mass is dark (Cowie et al. 


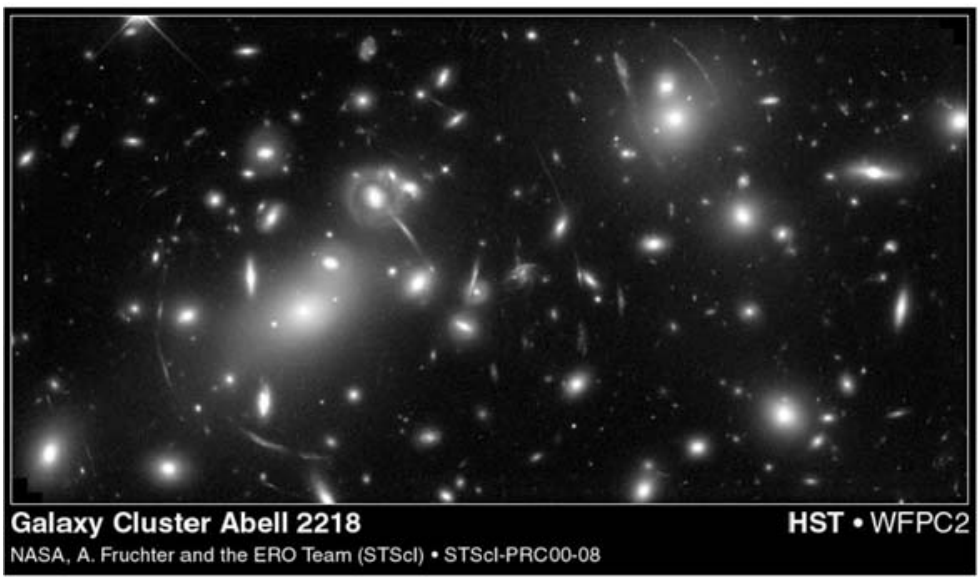

Fig. 2 HST optical image of Abell cluster A 2218. Many arcs and arclets are the images of background galaxies distorted by the gravitational field of the cluster (from http://antwrp.gsfc.nasa.gov/apod/ap011007.html)

1987), and the rest is almost all in the diffuse ICM component: galaxies only contribute a few percent to the total cluster mass, as inferred by Zwicky in 1937.

In this same 1937 paper, Zwicky also realized that one way to avoid the assumption of dynamical equilibrium when estimating the cluster mass is to use the deflection of light of background sources determined by the cluster gravitational potential well (Fig. 2); for an axially symmetric cluster, the total mass projected along the line of sight within the distance $r$ of closest approach of the light rays to the cluster centre is

$$
M(<r)=\frac{r c^{2}}{4 G} \alpha
$$

where $c$ is the speed of light, and $\alpha$ is the deflection angle (e.g. Schneider 2006). The first indication of a gravitational lensing effect in a galaxy cluster appeared fifty years later (Lynds and Petrosian 1986). To date, both strong gravitational lensing (Hennawi et al. 2008), which creates multiple images of background sources, and weak gravitational lensing (Dahle 2007), which produces small induced ellipticity in the shape of background galaxies, indicate that, if General Relativity is correct on the scales of clusters, $\sim 80-90 \%$ of the cluster mass is dark.

An alternative method to estimate the cluster mass, both in their virial region and their outskirts, which does not rely on the virial equilibrium assumption, is the caustic technique introduced by Diaferio and Geller (1997) and Diaferio (1999). This technique exploits the distribution of galaxies in redshift space to infer the galaxy escape velocity from the gravitational potential well of the cluster, and hence its mass. In fact, in the redshift diagram, namely line-of-sight velocity vs. projected distance $r$ from the cluster centre, cluster galaxies populate a trumpet-shaped region limited by two curves, named caustics, whose separation $\mathcal{A}(r)$ at each radius $r$ is proportional to the escape velocity. For a spherically symmetric cluster, the mass within radius $r$ is

$$
M(<r)=\frac{1}{2 G} \int_{0}^{r} \mathcal{A}^{2}(x) \mathrm{d} x
$$




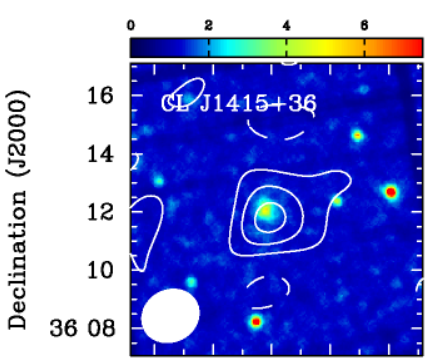

$141530 \quad 20 \quad 10 \quad 150 \quad 50$

Right Ascension (J2000)

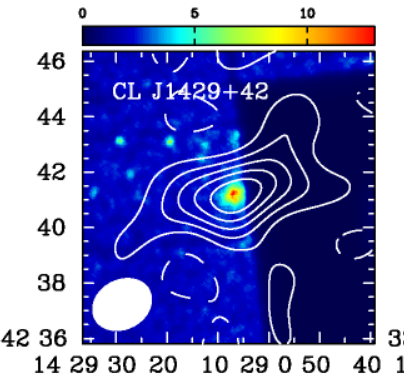

Right Ascension (J2000)

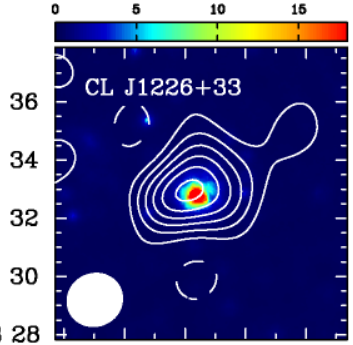

Right Ascension (J2000)

Fig. 3 Contours of the SZ effect measurements with SZA overlaid on the X-ray images (XMM EPIC/MOS) of the clusters ClJ1415.1 + $3612(z=1.03)$, ClJ1429.0 + $4241(z=0.92)$ and ClJ1226.9 $+3332(z=0.89)$. The filled ellipses show the FWHM of the SZ observations. See Muchovej et al. (2007) for details

All the mass estimation methods used to date, and described here, indicate that the dark matter contributes $\sim 80-90 \%$ of the total cluster mass, the ICM contributes $\sim 10-20 \%$, and the galaxies contribute less than a few percent. With the advance of X-ray spectroscopy, it has become clear that the ICM is chemically enriched to $\sim 0.5$ Solar abundances (Werner et al. 2008-Chap. 16, this issue): given the relative mass contribution of the cluster components, it follows that the total mass in metals in the ICM is larger than the sum of the mass in metals of the individual galaxies.

\subsubsection{Clusters as Probes for Cosmology}

Right after the detection of the X-ray emission from clusters, Sunyaev and Zeldovich (1972) realised that inverse Compton scattering of the Cosmic Microwave Background (CMB) photons by the free electrons of the ICM can produce secondary anisotropies in the CMB maps: $\Delta I_{\mathrm{CMB}} / I_{\mathrm{CMB}}=f(v) y$, where $f(v)$ is a spectral function and

$$
y=\int \frac{k T}{m_{\mathrm{e}} c^{2}} \sigma_{\mathrm{T}} n_{\mathrm{e}} \mathrm{d} l
$$

is the Comptonization parameter; here, $m_{\mathrm{e}}$ is the electron mass, $\sigma_{\mathrm{T}}$ the Thomson crosssection, and the integral is along the line of sight $l$. Typical ICM temperatures $k T$ and electron number densities $n_{\mathrm{e}}$ in clusters yield $\Delta I_{\mathrm{CMB}} / I_{\mathrm{CMB}} \approx 10^{-4}$ (Fig. 3). Only over the last few years the sensitivity of radio telescopes and the control of systematics have been sufficient to measure reliably this Sunyaev-Zeldovich (SZ) effect and its spectral signature in massive clusters (Bonamente et al. 2006). The major advantage of the SZ effect over other cluster observables is that the amplitude of the CMB fluctuation is independent of the cluster redshift $z$, because the CMB intensity increases as $(1+z)^{4}$ and compensates the $(1+z)^{-4}$ decrease of the SZ "surface brightness". This property has recently boosted an entire research field of cluster surveys with the SZ effect (Carlstrom et al. 2002). In fact, "flux-limited" SZ cluster surveys are almost mass-limited cluster surveys (Bartlett 2006). This property is extremely relevant, because the mass of clusters is a sensible cosmological probe, as we explain below.

If clusters are in virial equilibrium, we can derive simple relations between their global properties, namely mass, galaxy velocity dispersion, number of galaxies (richness), X-ray luminosity, ICM temperature, and so on. By considering for example the virial relation 
$3 k T /\left(2 \mu m_{\mathrm{p}}\right)=G M / R$, the scaling relation between the total mass $M$ and the gas temperature $k T$ reads

$$
k T=3.229\left(\frac{\mu}{0.6}\right)\left(\frac{\delta}{500}\right)^{1 / 3}\left(\frac{M}{10^{14} h^{-1} \mathrm{M}_{\odot}}\right)^{2 / 3} \mathrm{keV}
$$

where $\delta$ is the average cluster overdensity w.r.t. the critical density $3 H_{0}^{2} /(8 \pi G)$ of the universe, with $H_{0}=100 \mathrm{~km} \mathrm{~s}^{-1} \mathrm{Mpc}^{-1}$ being the Hubble constant at the present time. Quantities in (8) are normalized to typical cluster values. Analogously, we can write the total X-ray luminosity

$$
\begin{aligned}
L_{X}= & 1.327 \times 10^{43}\left(\frac{f_{\text {gas }}}{0.1 h^{-3 / 2}}\right)^{2}\left(\frac{0.6}{\mu}\right)\left(\frac{n}{10^{-3} h^{2} \mathrm{~cm}^{-3}}\right)\left(\frac{T}{\mathrm{keV}}\right)^{0.4} \\
& \times\left(\frac{M}{10^{14} h^{-1} \mathrm{M}_{\odot}}\right) h^{-2} \mathrm{erg} \mathrm{s}^{-1}
\end{aligned}
$$

where we approximated the cooling function $\Lambda(T)=0.843 \times 10^{-23}(\mathrm{kT} / \mathrm{keV})^{0.4} \mathrm{erg} \mathrm{cm}^{3} \mathrm{~s}^{-1}$ at $k T \gtrsim 1 \mathrm{keV}$, which holds for gas with poor metallicity, and assumed $n_{\mathrm{e}}=n_{\text {ions }} \equiv n=$ $f_{\text {gas }} \rho /\left(\mu m_{\mathrm{p}}\right)$, where $f_{\text {gas }}$ is the fraction of the cluster total mass in the ICM and $\rho$ is the cluster total mass density.

These scaling relations provide a straightforward method to estimate the cluster mass from a directly observable quantity, like the X-ray temperature. Quantities related to X-ray observations have received particular attention because of their robustness and the relatively simple physics involved in their correlations (Rosati et al. 2002).

Cluster masses are extremely relevant to constrain the cosmological model, because clusters populate the exponential tail of the mass function of galaxy systems. If the power spectrum of the primordial perturbations of the mass density field in the early universe is a power law with index $n$, the number per unit volume $[\mathrm{d} n(M) / \mathrm{d} M] \mathrm{d} M$ of galaxy systems with total mass in the range $(M, M+\mathrm{d} M)$ is (Press and Schechter 1974)

$$
\frac{\mathrm{d} n(M)}{\mathrm{d} M} \mathrm{~d} M=\frac{1}{\sqrt{\pi}} \frac{\bar{\rho}}{M^{2}}\left(1+\frac{n}{3}\right)\left(\frac{M}{M_{*}}\right)^{(n+3) / 6} \exp \left[-\left(\frac{M}{M_{*}}\right)^{(n+3) / 3}\right] \mathrm{d} M,
$$

where $\bar{\rho}$ is the (constant) comoving mean mass density of the universe and $M_{*}$ is a parameter depending on the normalisation $\sigma_{8}$ of the power spectrum and on the structure growth factor, which, in turn, depends on time, the cosmological density parameter $\Omega_{m}$ and the cosmological constant $\Omega_{\Lambda} . M_{*}$ increases with time when $n>-3$ and $M_{*} \sim 10^{14} h^{-1} \mathrm{M}_{\odot}$ at the present epoch. Clearly, since the exponential cut off dominates the mass function at $M \gtrsim M_{*}$, the evolution of the cluster number density is a very sensitive indicator of the power spectrum normalisation and of the cosmological parameters (Fig. 4). In practice, the application of this idea requires modern versions of the Press-Schechter mass function which are more sophisticated than (10) (Sheth and Tormen 1999); moreover, care must be taken to deal with the degeneracy among the cosmological parameters (e.g. Voit 2005), especially between $\Omega_{\mathrm{m}}$ and $\sigma_{8}$, which follow a relation of the form $\sigma_{8}=A \Omega_{\mathrm{m}}^{-\alpha}$, with $A \approx 0.5$ and $\alpha \approx 0.5$ (e.g. Reiprich and Böhringer 2002). Nevertheless, by simply assuming that the mean mass density of the universe must be smaller than the mean density within clusters and larger than the mean density computed including only the richest clusters, Abell already in 1965 estimated $\Omega_{\mathrm{m}} \approx 0.2$, a value remarkably close to the currently accepted estimate $\Omega_{\mathrm{m}}=0.26$ (Spergel et al. 2007). 
Fig. 4 Mass function of the HIFLUGCS X-ray clusters (dots with error bars). The solid line is the best fit with $\Omega_{\mathrm{m}}=0.12$ and $\sigma_{8}=0.98$. The dashed and dotted lines are the best fits with $\Omega_{\mathrm{m}}=0.5$ or $\Omega_{\mathrm{m}}=1.0$ held fixed, which yield $\sigma_{8}=0.60$ and $\sigma_{8}=0.46$, respectively. From Reiprich and Böhringer (2002)

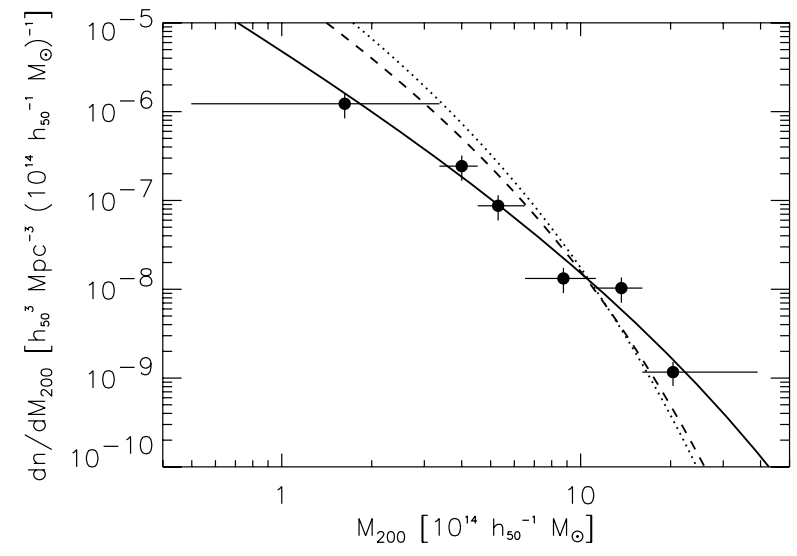

In most mass estimation techniques, the assumption of dynamical equilibrium is fundamental for obtaining accurate estimates. There is however overwhelming evidence that many clusters are out of equilibrium, as described in the following sections.

\subsection{Evidence of Non-equilibrium and Formation Processes}

\subsubsection{Cluster Galaxies}

Elliptical and lenticulars (early-type) galaxies tend to reside in high-density regions, whereas spiral (late-type) galaxies are more common in low-density regions. This morphology-density relation has been known since a publication of Shapley in 1926, and possibly earlier. The first statistical analysis based on a large sample of 55 clusters showed that, in the local universe, the fraction of spirals is $80 \%, 60 \%$ and $0 \%$ in the field, outskirts and cores of clusters, respectively (Dressler 1980). Subsequent work confirmed the connection between galaxy properties and local density: spirals in clusters are $\mathrm{H}$ I- and dust-deficient, have larger metallicity and show an excess of radio continuum emission (see for a review Boselli and Gavazzi 2006).

At higher redshifts $(z \sim 1)$, the fraction of spirals and irregulars in high-density regions increases at the expense of the fraction of lenticulars (Postman et al. 2005; Smith et al. 2005). Larger gas reservoirs and galaxy close encounters and collisions, which are more frequent in dense environments and favour the transformation of galaxy morphology, increase the star formation activity, as it occurs in starburst galaxies; indeed mid-infrared observations of luminous infrared galaxies show a more intense star formation activity in cluster environment at increasing redshift (Metcalfe et al. 2005; Bai et al. 2007). Although, on average, the properties of galaxies vary smoothly from the cluster centre to the outskirts, before reaching the average properties of galaxies in the field (e.g. Rines et al. 2005), the galaxy properties within individual clusters can show significant spatial variations, like the different slopes of the galaxy luminosity function in different regions of Coma (Adami et al. 2007).

In clusters with irregular morphology, the major axes of the bright galaxies tend to be aligned both with each other and with the major axis of the parent cluster (Plionis et al. 2003), as a century ago Wolf (1902) already pointed out for the galaxies in Coma. An alignment between the major axis of the bright central galaxy $(\mathrm{BCG})$ or the $\mathrm{cD}$ galaxy and the major axis of the parent cluster is also present, as first noticed by Carter and Metcalfe 
Fig. 5 Differential velocity dispersion profiles of galaxies brighter than $M_{R}=-20.5$ in the CNOC1 clusters

$(0.18<z<0.55)$. Top panel: Profiles of blue (open squares) and red (filled squares) galaxies; the rest-frame colour threshold is $B-V=0.85$. The dashed and the solid lines show the corresponding profiles of the blue and red galaxies in mock clusters simulated with a

$N$-body + semi-analytic technique (Diaferio et al. 2001; see Sect. 3). Bottom panel: Velocity dispersion profiles of disk-dominated galaxies (open squares) and bulge-dominated galaxies (filled squares). See Diaferio et al. (2001) for details

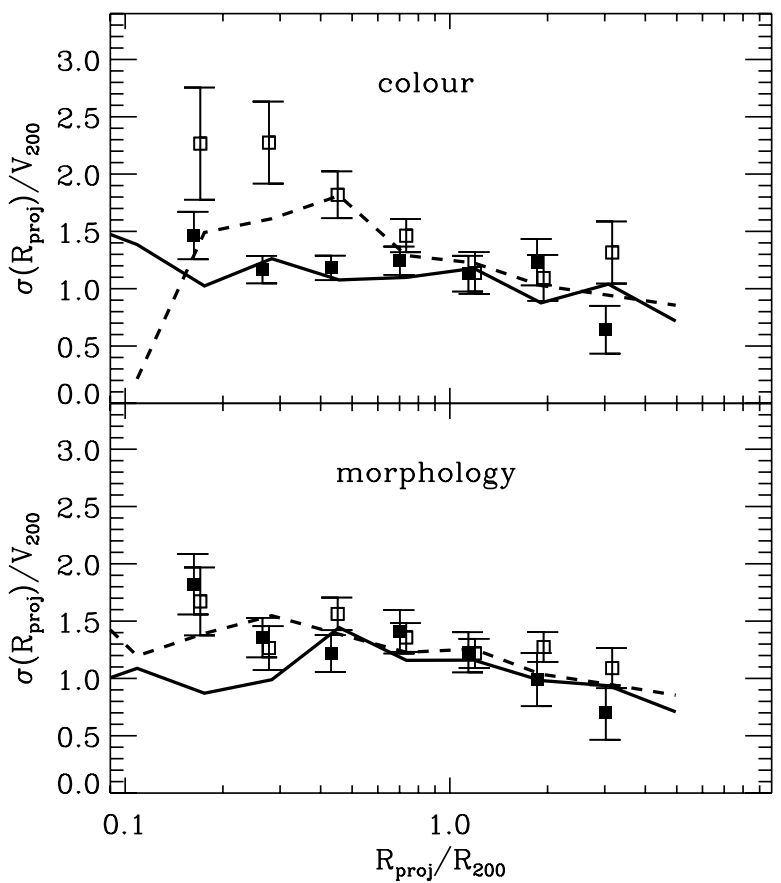

(1980). Any alignment present in the initial conditions is expected to be erased by multiple galaxy encounters occurring in the cluster environment. Therefore, the presence of alignment should indicate a young dynamical state of the cluster.

Blue cluster galaxies show larger velocity dispersions than red galaxies (Fig. 5; Biviano et al. 1997). This difference is attributed to more elongated orbits (Biviano and Katgert 2004), indicating that, unlike the red galaxies which are likely to be in dynamical equilibrium within the cluster gravitational potential well, blue galaxies are, on average, possibly falling onto the cluster for the first time.

Intracluster stars detected with planetary nebulae or as diffuse light in clusters at low (Gerhard et al. 2005) and intermediate redshift (Zibetti et al. 2005) can be the relics of tidal interactions suffered by falling galaxies (Covone et al. 2006) and/or the relics of merging of galaxies already in place in the forming gravitational potential well of the cluster (Murante et al. 2007), as observed in real clusters (Rines et al. 2007); intracluster stars can contain from 10 to $50 \%$ of the mass in stars in the core of clusters and can show distinct kinematic substructure (Arnaboldi et al. 2006).

\subsubsection{Substructure, Mass Accretion and Non-thermal Phenomena}

A large fraction of clusters shows the presence of substructure both in their galaxy distribution and in their X-ray emission morphology. The fraction of clusters with substructure depends on the cluster sample and on the substructure identification technique, but it is substantial, lying in the range 30-80\% (see Ramella et al. 2007, and references therein). The X-ray band also shows patchy temperature (Belsole et al. 2005) and metallicity maps (e.g. Hayakawa et al. 2006; Finoguenov et al. 2006, Fig. 6; see Werner et al. 2008-Chap. 16, this issue). Moreover, the X-ray morphology is increasingly irregular with increasing redshift (Jeltema et al. 2005). Where the angular resolution is low and detailed maps of the 

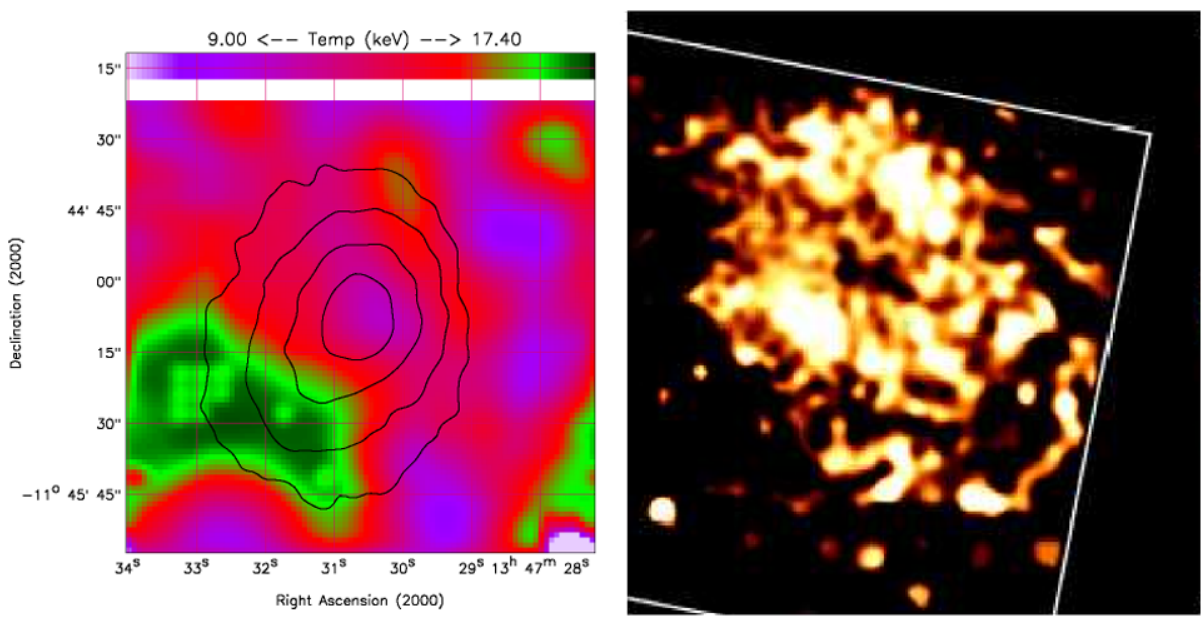

Fig. 6 Left panel: Temperature map of the cluster RX J1347.5-1145. The superposed contours show the X-ray surface brightness. See Gitti and Schindler (2004) for details. Right panel: Map of the metallicity abundance of the central region of A426, the Perseus cluster; brighter regions are more metal rich. See Sanders et al. (2005) for details

X-ray surface brightness cannot be obtained, a clear indication that, in some clusters, the ICM might be out of equilibrium comes from the hard, possibly thermal, excess (Rephaeli et al. 2008-Chap. 5, this issue), which appears when the X-ray spectrum is fitted assuming a plasma with single temperature and metallicity. In these cases, two or more temperatures are required to yield a reasonable fit.

All these pieces of evidence suggest that clusters accrete matter from the surrounding regions. The cluster 1ES 0657-55.8, dubbed "the bullet cluster" (Markevitch et al. 2002; Barrena et al. 2002), is a spectacular example of a merging cluster at intermediate redshift $(z=0.296)$. At low redshifts, there are many other examples of merging clusters or galaxy groups falling onto clusters along filamentary structure. To mention a few: Coma (Colless and Dunn 1996; Adami et al. 2005), A 521 (Ferrari et al. 2003), A 754 (Henry et al. 2004), A 2199 (Rines et al. 2001), A 2219 (Boschin et al. 2004), A 3560 in the Shapley supercluster (Bardelli et al. 2002), A 3921 (Ferrari et al. 2005). At high redshift the number of irregular and merging clusters increases substantially (Rosati 2004; Kodama et al. 2007).

Accretion of matter is a natural explanation for the patchy morphology of the X-ray emission and metallicity maps; it can also cause most of the turbulence in the ICM (Schuecker et al. 2004) and generate shocks (Bykov et al. 2008a-Chap. 7, this issue), which can be efficient generators of high-energy cosmic rays.

Non-thermal processes in the ICM appear in the X-ray and radio bands (Rephaeli et al. 2008-Chap. 5, this issue). Non-thermal hard X-ray emission is indeed observed in a few systems (e.g. Petrosian et al. 2006; Fusco-Femiano et al. 2007, and references therein). However, the existence of both the hard X-ray emission excess (Rossetti and Molendi 2004) and the soft X-ray and UV emission excesses, regardless of their thermal or non-thermal origin, are still debated (e.g. Lieu and Mittaz 2005; Nevalainen et al. 2007; Werner et al. 2007; Durret et al. 2008-Chap. 4, this issue).

At radio frequencies, the cluster emission can be both extended and associated with galaxies. One can infer the direction of the bulk motion of gas clouds within the ICM from 
the deviations that the ICM kinetic pressure exerts on the radio jets emerging from active galactic nuclei (AGN) (Burns et al. 2002).

The extended radio emission has a steep spectrum and can appear with two different morphologies: either the emission comes from the cluster centre (halo), is extended and regular, or the emission comes from the cluster outskirts and is elongated (relic) (Feretti and Giovannini 2007; Ferrari et al. 2008-Chap. 6, this issue). By combined radio and hard X-ray observations or by Faraday rotation measurements one can infer the presence of magnetic fields in the ICM with intensity ranging from a few tenths to several $\mu$ Gauss (e.g. Blasi et al. 2007; Ferrari et al. 2008-Chap. 6, this issue).

Both the radio and the hard X-ray emissions originate from relativistic electrons: the former is synchrotron radiation, the latter is emission due to inverse Compton scattering with the CMB photons (Petrosian et al. 2008-Chap. 10, this issue). Among the many acceleration mechanisms proposed to produce the relativistic electrons (Petrosian and Bykov 2008-Chap. 11, this issue), shocks due to the accretion of matter from the surrounding regions seem to be one of the most efficient.

\subsection{Connection with the Large-Scale Structure}

\subsubsection{A Morphology-Density Relation for Clusters}

It has been known for a long time that clusters are not randomly distributed in the universe but form large concentrations named superclusters (Shapley 1933). The connection between clusters and the large-scale structure has become evident with extended galaxy redshift surveys, like the CfA (de Lapparent et al. 1986), the SDSS (York et al. 2000), and the $2 \mathrm{dF}$ (Colless et al. 2001) redshift surveys.

To quantify the distribution of objects in space, the simplest quantity to compute is the two-point correlation function $\xi(r)$, which yields the probability of finding two objects at separation $r$ in excess to a Poisson distribution. Clusters correlate according to the correlation function $\xi=\left(r / r_{0}\right)^{\gamma}$, with $\gamma \approx-1.8$ (Bahcall and Soneira 1983). The correlation length $r_{0}$ is proportional to the cluster richness: richer clusters are rarer and their mean intercluster separation $d$ larger. One finds $r_{0} \propto d^{0.5}$, and $r_{0} \approx 20-25 h^{-1} \mathrm{Mpc}$ for rich clusters. However, this $r_{0}-d$ relation cannot be fully responsible for the large range, from $\sim 10$ to $\sim 30 h^{-1} \mathrm{Mpc}$, covered by the correlation length $r_{0}$ when one considers clusters with increasing substructure (Fig. 7; Plionis and Basilakos 2002). Moreover, cluster shapes are generally elongated, as first quantified by Sastry (1968), and close clusters have their major axes aligned (Binggeli 1982). In the APM cluster sample, containing 903 objects, this alignment is stronger when the fraction of substructure is larger (Plionis and Basilakos 2002).

This connection between the properties of clusters and their large-scale environment is also apparent in the X-ray band: the X-ray luminosity of clusters (Böhringer 2004) and the irregularity of the X-ray surface brightness maps (Schuecker et al. 2001) are larger in environments with a higher cluster number density.

These features appear in a scenario (West 1994) where clusters form by episodic mass accretion along preferential directions (Colberg et al. 1999), in agreement with the currently accepted model of the formation of cosmic structure (see Sect. 3). Future estimates of cluster peculiar velocities based on the kinetic SZ effect, when corrected of their systematics (Diaferio et al. 2005), will further test whether the correlation between the evolution of the peculiar velocity and the local density predicted by this model (Diaferio et al. 2000; Sheth and Diaferio 2001) is correct. 
Fig. 7 Two-point correlation function of the 903 APM cluster sample (open circles) and of a subsample of clusters with significant substructure (filled circles). Insert: Correlation length $r_{0}$ vs. the significance level $\sigma$ of substructure. See Plionis and Basilakos (2002) for details

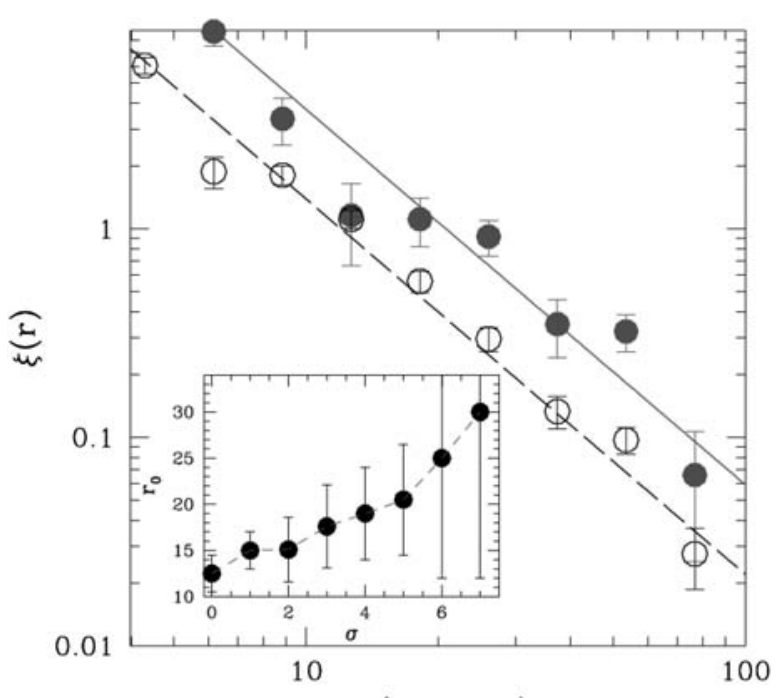

\subsubsection{The Surrounding WHIM}

The optical depth of the intergalactic medium at $z \gtrsim 2$ derived from the Lyman- $\alpha$ forest of QSO spectra (Rauch et al. 1997), the abundances of light elements combined with the predictions of the standard Big Bang nucleosynthesis model and the power spectrum of the CMB (Spergel et al. 2007) are all consistent with a density of baryonic matter $\Omega_{\mathrm{b}} h^{2} \simeq 0.022$. At low redshift, baryonic matter within galaxies and within the diffuse hot medium of galaxy clusters and groups only accounts for $\sim 15 \%$ of this $\Omega_{\mathrm{b}}$ (Fukugita et al. 1998).

The hierarchical clustering model solves this discrepancy by predicting that $50 \%$ of the baryons permeating the matter distribution is in a warm phase with $k T$ in the range $0.01-1 \mathrm{keV}$, overdensity in the range $0.1-10^{4}$ and median $\sim 10-20$, and median metallicity $\sim 0.2$ Solar, with a large spread, for oxygen (Cen and Ostriker 1999, 2006; Bertone et al. 2008-Chap. 14, this issue). The WHIM overdensity increases with increasing dark matter overdensity. Therefore, the outskirts of clusters and their environments are expected to be sources of a thermal soft X-ray emission. However, both the expected low surface brightness of the WHIM and the intergalactic and Galactic absorption make this observation very challenging. Nevertheless, there are claims of detection in many clusters, including Coma (Takei et al. 2007). In fact, rather than detecting the thermal X-ray emission (Durret et al. 2008Chap. 4, this issue) directly, the most promising technique is searching for thermal UV and X-ray absorption lines in the spectra of bright background quasars or blazars (Richter et al. 2008-Chap. 3, this issue). Even with this strategy, current instruments (Paerels et al. 2008-Chap. 19, this issue) must be used at their sensitivity limits and the most plausible detections (e.g. Nicastro et al. 2005) are indeed still debated (Kaastra et al. 2006). Firm detections are however of great relevance, because the existence of the WHIM and the estimate of its properties would be an extraordinary confirmation of the current model of structure formation. 


\section{The Modelling Framework}

\subsection{Players: Gravity and Beyond}

Modelling the formation and evolution of galaxy clusters requires the set-up of a complete cosmological context. According to the current paradigm of structure formation (as reviewed, e.g., in Springel et al. 2006), inflation amplifies quantum fluctuations, which are present in the dark matter density field of the early universe, to cosmic scales; at the time of matter and radiation density equality, these fluctuations start growing by gravitational instability. After radiation and ordinary baryonic matter decouple, radiation pressure stops supporting the baryonic density perturbations against self-gravity and against the pull of the dark matter gravitational potential wells that have already formed: the dark matter inhomogeneities thus accelerate the collapse of ordinary matter.

The pre-inflationary Gaussian quantum fluctuations generate a scale-free power spectrum of the primordial density perturbations. At later times, the dark matter particles, which have sufficient momenta to stream out of the denser regions, set in a characteristic scale in the power spectrum, which is proportional to the typical velocity of the dark matter particles themselves; below this scale the density perturbations are damped. The existence of quasars and galaxies at high redshift (Fan et al. 2004), the power spectrum of the CMB (Smoot et al. 1992; de Bernardis et al. 2000; Hanany et al. 2000), and the power spectrum of the largescale distribution of galaxies (Tegmark et al. 2004) all agree with the hypothesis that the dark matter is "cold", namely that the dark matter particles become non-relativistic at very early times and the damping scale is $\ll 1 h^{-1}$ Mpc. In these Cold Dark Matter (CDM) models, larger systems form by the aggregation of smaller systems and galaxy clusters are the nodes of the network of sheets and filaments which constitutes the matter density field (Fig. 8). Clusters can form at reasonably recent times, with the actual formation time depending on the cosmological parameters.

In CDM models, the scale-free properties of the primordial power spectrum are preserved on scales larger than the scales of galaxies. This feature implies that the dark matter halos of galaxies, groups and clusters evolve self-similarly. If gravitation is the only force playing,

Fig. 8 Map density at $z=0$ of the gas in a slice of the $N$-body/hydrodynamics simulation of Borgani et al. (2004). The volume shown is $192 h^{-1} \mathrm{Mpc}$ on a side and $12 h^{-1} \mathrm{Mpc}$ thick. The high-density regions are white, the low-density regions dark. See Borgani et al. (2004) for details

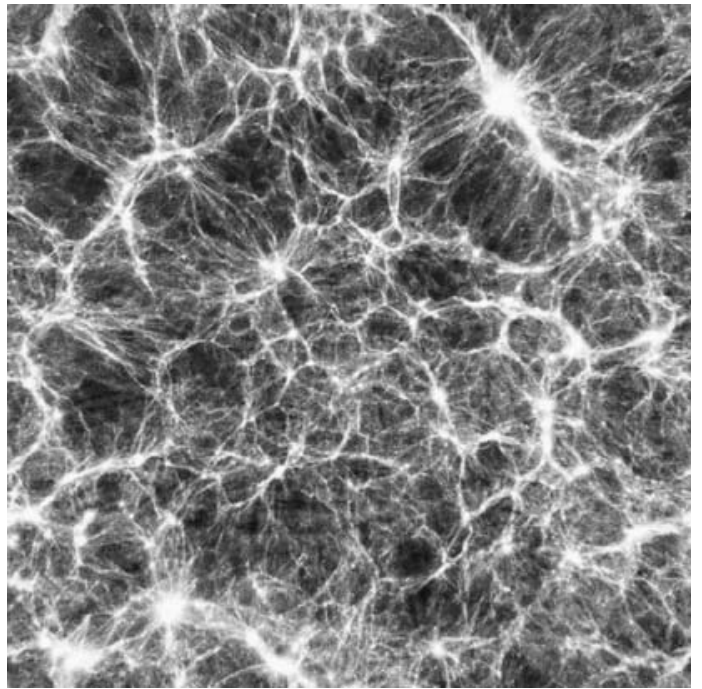


the physics of the ICM is ruled by adiabatic compression and shock heating, and we can derive simple relations between cluster properties at different redshifts (Kaiser 1986). The cluster virial mass scales as $M_{\mathrm{vir}} \propto \rho_{\mathrm{c}}(z) \Delta_{\mathrm{c}}(z) R^{3}$, where $R$ is the cluster size in physical units, $\rho_{\mathrm{c}}(z)=3 H^{2}(z) /(8 \pi G)$ is the critical density of the universe, and $\Delta_{\mathrm{c}}(z)$ is the cluster density in units of $\rho_{\mathrm{c}}(z) ;{ }^{2} \rho_{\mathrm{c}}(z)$ scales with redshift $z$ as $\rho_{\mathrm{c}}(z) \propto E^{2}(z)=\Omega_{\mathrm{m}}(1+z)^{3}+$ $\left(1-\Omega_{\mathrm{m}}-\Omega_{\Lambda}\right)(1+z)^{2}+\Omega_{\Lambda}$. The cluster size thus scales as $R \propto M_{\mathrm{vir}}^{1 / 3} \Delta_{\mathrm{c}}^{-1 / 3}(z) E^{-2 / 3}(z)$, and the temperature as $T \propto M_{\mathrm{vir}} / R \propto M_{\mathrm{vir}}^{2 / 3} \Delta_{\mathrm{c}}^{1 / 3}(z) E^{2 / 3}(z)$; thus, for example, one finds for the X-ray luminosity due to Bremsstrahlung emission:

$$
L_{X} \propto \int n_{\mathrm{e}} n_{\text {ions }} \Lambda(T) \mathrm{d} V \propto M_{\mathrm{vir}}^{2} R^{-3} T^{1 / 2} \propto M_{\mathrm{vir}}^{4 / 3} E^{7 / 3}(z) \Delta_{\mathrm{c}}^{7 / 6}(z)
$$

or for the Comptonization parameter

$$
y \propto \int n_{\mathrm{e}} T \mathrm{~d} l \propto M_{\mathrm{vir}} R^{-2} T \propto M_{\mathrm{vir}} E^{2}(z) \Delta_{\mathrm{c}}(z) .
$$

These scaling relations only partially agree with observations; in fact, (1) the relative contribution of non-gravitational sources (AGN, galactic winds, supernovae, turbulence) to the ICM energy budget depends on the mass of the cluster and affects the slope of the scaling relations; and (2) the merging history of the cluster introduces an intrinsic scatter in the relations (Cavaliere et al. 1998). Moreover, clusters at high redshift have scaling relations steeper than the self-similar prediction (Ettori et al. 2004).

The deviations from the self-similar scaling relations are clearly related to non-gravitational processes. The interplay between gravitational and non-gravitational processes is highly non-linear and treating it appropriately requires sophisticated modelling. Over the last thirty years two main approaches have been followed: numerical simulations (Dolag et al. 2008a-Chap. 12, this issue) and an analytic (or semi-analytic) approach. Over the last ten years, the two approaches have been combined.

\subsection{Making Clusters: Recipes, Ingredients and Tools}

For the last thirty years, numerical simulations have represented the main tool of theoretical astrophysicists to investigate the non-linear evolution of cosmic structure and compare the theoretical results with observations. The first computational device for simulating galaxy interactions used an ingenious system of light-bulbs (Holmberg 1941). Numerical simulations of astrophysical systems have now reached tremendous sophistication (Dolag et al. 2008a-Chap. 12, this issue) when compared to the first numerical integration of the equations of motion of a few stars in a star cluster (von Hoerner 1960). Current numerical models reproduce the gross features of the large-scale structure, and the distribution of galaxies and diffuse baryons (Borgani et al. 2008a-Chap. 13; Bertone et al. 2008-Chap. 14, this issue), including the statistical properties of the Ly $\alpha$ forest (Springel et al. 2006).

Until the 1980s, numerical simulations relied on $N$-body techniques only to follow the evolution of the dissipationless component of cosmic structure. Particles in the simulations were first identified with galaxies and later on with dark matter particle tracers.

\footnotetext{
${ }^{2} \mathrm{~A}$ well-known approximation is

$$
\Delta_{\mathrm{c}}(z)=18 \pi^{2}+ \begin{cases}60 w-32 w^{2}, & \Omega_{\mathrm{m}} \leq 1, \Omega_{\Lambda}=0 \\ 82 w-39 w^{2}, & \Omega_{\mathrm{m}}+\Omega_{\Lambda}=1\end{cases}
$$
}

where $w=\Omega_{\mathrm{m}}(z)-1$ (Bryan and Norman 1998). 
Peebles (1970) and White (1976) were the first to simulate the formation of a galaxy cluster with vacuum boundary conditions. To obtain a realistic distribution of matter, however, it is necessary to simulate a cosmological volume, with periodic boundary conditions, which is large enough to be representative of the universe. The first simulation of this kind, with a cold dark matter power spectrum of the initial density perturbations, demonstrated that dark matter must be constituted of cold collisionless particles, rather than massive neutrinos (Davis et al. 1985). Recent dark matter only simulations yield clusters whose number density evolution (Evrard et al. 2002), virial relations (Evrard et al. 2008), and connection between their shape and the large-scale structure (Altay et al. 2006; Lee and Evrard 2007) resemble the observations and can be used as probes of the cosmological model.

In the late 1980s, smoothed particle hydrodynamics (SPH) (Evrard 1988; Hernquist and Katz 1989) and mesh techniques (Cen et al. 1990) were introduced to follow the evolution of the dissipative component. The presence of dissipative gas substantially increases the number of physical processes to be taken into account and therefore increases the complexity of the simulations. In fact, a priori, it is not guaranteed that the different techniques yield similar results and some code comparisons have been performed over the years to check the consistency of the various integration schemes (Kang et al. 1994; Frenk et al. 1999; O'Shea et al. 2005).

The simplest approximation is to consider the gas non-radiative and perform adiabatic simulations (Evrard 1990). This model is able to reproduce the general trend of the selfsimilar scaling laws, but disagrees with observations in detail, yielding, in general, shallower X-ray luminosity-temperature and mass-temperature relations (Navarro et al. 1995b). To reproduce the observations more closely, and explain the deviations from the self-similar model, radiative cooling (Thomas and Couchman 1992; Katz and White 1993) and nongravitational heating processes (Navarro et al. 1995b) must be included. More realistic models, attempting to reproduce the thermal properties of the ICM and the stellar content of clusters, have to include explicitly all those intimately coupled processes which rule galaxy formation and the thermodynamics of the ICM: star formation, energy feedback from supernovae explosion (Valdarnini 2002) and galactic winds (Metzler and Evrard 1994; Dolag et al. 2008a-Chap. 12; Borgani et al. 2008a-Chap. 13, this issue), besides metal production and chemical enrichment (Valdarnini 2003; Schindler and Diaferio 2008Chap. 17; Borgani et al. 2008b-Chap. 18, this issue), which strongly affects the gas cooling rate; moreover, one needs to include magnetic fields and its associated non-thermal processes (Dolag et al. 1999, 2008b-Chap. 15, this issue).

The galaxy population in the simulated clusters have shown some rough similarities with observations since the very first numerical experiments (Evrard et al. 1994); many other observed properties are reproduced at different levels of agreement, including the soft X-ray thermal emission (Cheng et al. 2005). Nevertheless, in no simulation today the thermodynamical properties of the ICM, the stellar mass fraction and the galaxy luminosity function simultaneously match the observations. One of the most serious discrepancies with observations is the overproduction of stars: the fraction of baryons in stars usually is $\sim 20 \%$ of the total baryon mass in clusters, at least a factor of two larger than observed (Borgani et al. 2004). A possible solution has been identified in the inhibition of the ICM cooling by the energy injection of AGN jets, as suggested by the X-ray cavities in correspondence of the radio emission observed around cluster central galaxies (McNamara et al. 2006; McNamara and Nulsen 2007); the presence of these cavities correlates with the X-ray temperature drops in the centre of some clusters, early interpreted as cooling flows (Dunn and Fabian 2006); however, the details of the heating mechanism by AGN jets remain uncertain (Voit 2005). 
The main difficulty when treating most of the dissipative processes resides in the fact that they occur on scales much smaller than the spatial resolution of the simulation and must be approximated by phenomenological recipes: gas cooling, star formation and stellar evolution, which occur at scales $\ll 1 \mathrm{pc}$, have effects on galactic and extragalactic scales $\gg 1 \mathrm{Mpc}$; thus the simulations should model a spatial range of at least six orders of magnitude. These large dynamic ranges can be covered by neither a single numerical experiment nor experiments which rely on the resimulation technique, where an individual cluster extracted from a large-scale cosmological simulation is resimulated at a much higher resolution with appropriate boundary conditions (Navarro et al. 1995a).

The large number of physical processes and the large computational resources required to run an $\mathrm{N}$-body/hydrodynamics simulation for a realistic modelling of cluster and galaxy formation have suggested a complementary strategy to explore more efficiently the parameter space: the combination of semi-analytic models with merger trees of dark matter halos extracted from dissipationless $N$-body simulations (Roukema et al. 1997; Kauffmann et al. 1999). This approach has reproduced the evolution and present-day spatial and kinematic distribution of galaxies in the universe and in clusters (Springel et al. 2005). The major shortcoming of this strategy is that severe uncertainties in the predictions can be introduced by the crude simplification of the physical processes and especially of the thermodynamics of the ICM, usually assumed to be isothermal and in hydrostatic equilibrium within each dark matter halo. Some improvements can be introduced by describing the ICM thermal properties in terms of entropy generation and distribution (McCarthy et al. 2007), but much work remains to be done.

Acknowledgements The authors thank ISSI (Bern) for support of the team "Non-virialized X-ray components in clusters of galaxies". Partial support from the PRIN2006 grant "Costituenti fondamentali dell'Universo" of the Italian Ministry of University and Scientific Research and from the INFN grant PD51 is gratefully acknowledged. The authors also acknowledge financial support by the Austrian Science Foundation (FWF) through grants P18523-N16 and P19300-N16, by the Tiroler Wissenschaftsfonds and through the UniInfrastrukturprogramm 2005/06 by the BMWF.

\section{References}

G.O. Abell, Astrophys. J. Suppl. Ser. 3, 211 (1958)

G.O. Abell, Annu. Rev. Astron. Astrophys. 3, 1 (1965)

C. Adami, A. Biviano, F. Durret, A. Mazure, Astron. Astrophys. 443, 17 (2005)

C. Adami, F. Durret, A. Mazure et al., Astron. Astrophys. 462, 411 (2007)

G. Altay, J.M. Colberg, R.A.C. Croft, Mon. Not. R. Astron. Soc. 370, 1422 (2006)

V.A. Ambartsumian, Astron. J. 66, 536 (1961)

M. Arnaboldi, O. Gerhard, K.C. Freeman et al., in Planetary Nebulae in Our Galaxy and Beyond, ed. by M.J. Barlow, R.H. Méndez. Proc. IAU Symp. 234 (Cambridge Univ. Press, Cambridge, 2006), p. 337

N.A. Bahcall, R.M. Soneira, Astrophys. J. 270, 20 (1983)

L. Bai, D. Marcillac, G.H. Rieke et al., Astrophys. J. 664, 181 (2007)

S. Bardelli, T. Venturi, E. Zucca et al., Astron. Astrophys. 396, 65 (2002)

R. Barrena, A. Biviano, M. Ramella, E.E. Falco, S. Seitz, Astron. Astrophys. 386, 816 (2002)

J.G. Bartlett, astro-ph/0606241 (2006)

E. Belsole, J.-L. Sauvageot, G.W. Pratt, H. Bourdin, Adv. Space Res. 36, 630 (2005)

S. Bertone, J. Schaye, K. Dolag, Space Sci. Rev. (2008). doi:10.1007/s11214-008-9318-3

B. Binggeli, Astron. Astrophys. 107, 338 (1982)

A. Biviano, Untangling Coma Berenices: A new vision of an old cluster, in Proc. Meeting Held in Marseilles (France), June 17-20, 1997, ed. by A. Mazure, F. Casoli, F. Durret, D. Gerbal (Word Scientific, Singapore, 1998), p. 1

A. Biviano, From Messier to Abell: 200 years of science with galaxy clusters, in Constructing the Universe with Clusters of Galaxies, ed. by F. Durret, D. Gerbal. IAP 2000 meeting, astro-ph/0010409 (2000) 
A. Biviano, in Proc. of the XLI Rencontres de Moriond, ed. by L. Tresse, S. Maurogordato, J. Tran Thanh Van. XXVI Astrophysics Moriond Meeting: From dark halos to light, Editions Frontieres (2006)

A. Biviano, P. Katgert, Astron. Astrophys. 424, 779 (2004)

A. Biviano, P. Katgert, A. Mazure et al., Astron. Astrophys. 321, 84 (1997)

P. Blasi, S. Gabici, G. Brunetti, Int. J. Mod. Phys. A22, 681 (2007)

H. Böhringer, The large-scale environment of groups and clusters of galaxies, in Outskirts of Galaxy Clusters: Intense Life in the Suburbs, ed. by A. Diaferio. IAU Coll. 195 (2004), p. 12

M. Bonamente, M.K. Joy, S.J. LaRoque et al., Astrophys. J. 647, 25 (2006)

S. Borgani, G. Murante, V. Springel et al., Mon. Not. R. Astron. Soc. 348, 1078 (2004)

S. Borgani, A. Diaferio, K. Dolag, S. Schindler, Space Sci. Rev. (2008a). doi:10.1007/s11214-008-9317-4

S. Borgani, D. Fabjan, L. Tornatore et al., Space Sci. Rev. (2008b). doi:10.1007/s11214-008-9322-7

W. Boschin, M. Girardi, R. Barrena et al., Astron. Astrophys. 416, 839 (2004)

A. Boselli, G. Gavazzi, Publ. Astron. Soc. Pac. 118, 517 (2006)

G.L. Bryan, M.L. Norman, Astrophys. J. 495, 80 (1998)

J.O. Burns, C. Loken, K. Roettiger et al., New Astron. Rev. 46, 135 (2002)

A.M. Bykov, K. Dolag, F. Durret, Space Sci. Rev. (2008a). doi:10.1007/s11214-008-9312-9

A.M. Bykov, F.B.S. Paerels, V. Petrosian, Space Sci. Rev. (2008b). doi:10.1007/s11214-008-9309-4

J.E. Carlstrom, G.P. Holder, E.D. Reese, Annu. Rev. Astron. Astrophys. 40, 643 (2002)

D. Carter, N. Metcalfe, Mon. Not. R. Astron. Soc. 191, 325 (1980)

A. Cavaliere, N. Menci, P. Tozzi, Astrophys. J. 501, 493 (1998)

R. Cen, J.P. Ostriker, Astrophys. J. 514, 1 (1999)

R. Cen, J.P. Ostriker, Astrophys. J. 650, 560 (2006)

R.Y. Cen, J.P. Ostriker, A. Jameson, F. Liu, Astrophys. J. 362, L41 (1990)

L.-M. Cheng, S. Borgani, P. Tozzi et al., Astron. Astrophys. 431, 405 (2005)

J.M. Colberg, S.D.M. White, A. Jenkins, F.R. Pearce, Mon. Not. R. Astron. Soc. 308, 593 (1999)

M. Colless, A.M. Dunn, Astrophys. J. 458, 435 (1996)

M. Colless, G. Dalton, S. Maddox et al., Mon. Not. R. Astron. Soc. 328, 1039 (2001)

G. Covone, C. Adami, F. Durret et al., Astron. Astrophys. 460, 381 (2006)

L.L. Cowie, M. Henriksen, R. Mushotzky, Astrophys. J. 317, 593 (1987)

H. Dahle, astro-ph/0701598 (2007)

M. Davis, G. Efstathiou, C.S. Frenk, S.D.M. White, Astrophys. J. 292, 371 (1985)

P. de Bernardis, P.A.R. Ade, J.J. Bock et al., Nature 404, 955 (2000)

V. de Lapparent, M.J. Geller, J.P. Huchra, Astrophys. J. 302, L1 (1986)

A. Diaferio, Mon. Not. R. Astron. Soc. 309, 610 (1999)

A. Diaferio, M.J. Geller, Astrophys. J. 481, 633 (1997)

A. Diaferio, R.A. Sunyaev, A. Nusser, Astrophys. J. 533, L71 (2000)

A. Diaferio, G. Kauffmann, M.L. Balogh et al., Mon. Not. R. Astron. Soc. 323, 999 (2001)

A. Diaferio, S. Borgani, L. Moscardini et al., Mon. Not. R. Astron. Soc. 356, 1477 (2005)

K. Dolag, M. Bartelmann, H. Lesch, Astron. Astrophys. 348, 351 (1999)

K. Dolag, S. Borgani, S. Schindler, A. Diaferio, A.M. Bykov, Space Sci. Rev. (2008a). doi:10.1007/s11214008-9316-5

K. Dolag, A.M. Bykov, A. Diaferio, Space Sci. Rev. (2008b). doi:10.1007/s11214-008-9319-2

A. Dressler, Astrophys. J. 236, 351 (1980)

R.J.H. Dunn, A.C. Fabian, Mon. Not. R. Astron. Soc. 373, 959 (2006)

F. Durret, J.S. Kaastra, J. Nevalainen, T. Ohashi, N. Werner, Space Sci. Rev. (2008). doi:10.1007/s11214008-9313-8

S. Ettori, P. Tozzi, S. Borgani, P. Rosati, Astron. Astrophys. 417, 13 (2004)

A.E. Evrard, Mon. Not. R. Astron. Soc. 235, 911 (1988)

A.E. Evrard, Astrophys. J. 363, 349 (1990)

A.E. Evrard, F.J. Summers, M. Davis, Astrophys. J. 422, 11 (1994)

A.E. Evrard, T.J. MacFarland, H.M.P. Couchman et al., Astrophys. J. 573, 7 (2002)

A.E. Evrard, J. Bialek, M. Busha et al., Astrophys. J. 672, 122 (2008)

X. Fan, J.F. Hennawi, G.T. Richards et al., Astron. J. 128, 515 (2004)

J.E. Felten, R.J. Gould, W.A. Stein, N.J. Woolf, Astrophys. J. 146, 955 (1966)

L. Feretti, G. Giovannini, in Panchromatic View of Clusters of Galaxies and the Large-Scale Structure, ed. by M. Plionis, O. Lopez-Cruz, D. Hughes. Springer Lect. Notes in Phys. (Springer, Berlin, 2007 in press). (astro-ph/0703494)

C. Ferrari, S. Maurogordato, A. Cappi, C. Benoist, Astron. Astrophys. 399, 813 (2003)

C. Ferrari, F. Govoni, S. Schindler, A. Bykov, Y. Rephaeli, Space Sci. Rev. (2008). doi:10.1007/s11214008-9311-x

C. Ferrari, C. Benoist, S. Maurogordato, A. Cappi, E. Slezak, Astron. Astrophys. 430, 19 (2005) 
A. Finoguenov, M.J. Henriksen, F. Miniati, U.G. Briel, C. Jones, Astrophys. J. 643, 790 (2006)

C.S. Frenk, S.D.M. White, P. Bode et al., Astrophys. J. 525, 554 (1999)

M. Fukugita, C.J. Hogan, P.J.E. Peebles, Astrophys. J. 503, 518 (1998)

R. Fusco-Femiano, R. Landi, M. Orlandini, Astrophys. J. 654, L9 (2007)

O. Gerhard, M. Arnaboldi, K.C. Freeman et al., Astrophys. J. 621, L93 (2005)

M. Girardi, G. Giuricin, F. Mardirossian, M. Mezzetti, W. Boschin, Astrophys. J. 505, 74 (1998)

M. Gitti, S. Schindler, Astron. Astrophys. 427, L9 (2004)

H. Gursky, R. Levinson, E. Kellogg et al., Astrophys. J. 173, L99 (1972)

S. Hanany, P. Ade, A. Balbi et al., Astrophys. J. 545, L5 (2000)

A. Hayakawa, A. Hoshino, M. Ishida et al., Publ. Astron. Soc. Jpn. 58, 695 (2006)

J.F. Hennawi, M.D. Gladders, M. Oguri et al., Astron. J. 135, 664 (2008)

J.P. Henry, A. Finoguenov, U.G. Briel, Astrophys. J. 615, 181 (2004)

L. Hernquist, N. Katz, Astrophys. J. Suppl. Ser. 70, 419 (1989)

E. Holmberg, Astrophys. J. 94, 385 (1941)

T.E. Jeltema, C.R. Canizares, M.W. Bautz, D.A. Buote, Astrophys. J. 624, 606 (2005)

J.S. Kaastra, N. Werner, J.W.A. den Herder et al., Astrophys. J. 652, 189 (2006)

J.S. Kaastra, F.B.S. Paerels, F. Durret, S. Schindler, P. Richter, Space Sci. Rev. (2008). doi:10.1007/ s11214-008-9310-y

N. Kaiser, Mon. Not. R. Astron. Soc. 222, 323 (1986)

H. Kang, J.P. Ostriker, R. Cen et al., Astrophys. J. 430, 83 (1994)

N. Katz, S.D.M. White, Astrophys. J. 412, 455 (1993)

G. Kauffmann, J.M. Colberg, A. Diaferio, S.D.M. White, Mon. Not. R. Astron. Soc. 303, 188 (1999)

T. Kodama, M. Tanaka, I. Tanaka, M. Kajisawa, in Galaxy Evolution Across the Hubble Time, ed. by F. Combes, J. Palous. IAU Symp. 235 (2007), p. 170

J. Lee, A.E. Evrard, Astrophys. J. 657, 30 (2007)

R. Lieu, J.P.D. Mittaz, The Cluster soft excess: new faces of an old enigma, in The Identification of Dark Matter, ed. by N.J.C. Spooner, V. Kudryavtsev (World Scientific, Singapore, 2005), p. 18

R. Lynds, V. Petrosian, Bull. Am. Astron. Soc. 18, 1014 (1986)

M. Markevitch, A.H. Gonzalez, L. David et al., Astrophys. J. 567, L27 (2002)

I.G. McCarthy, R.G. Bower, M.L. Balogh et al., Mon. Not. R. Astron. Soc. 376, 497 (2007)

B.R. McNamara, P.E.J. Nulsen, Annu. Rev. Astron. Astrophys. 45, 117 (2007)

B.R. McNamara, D.A. Rafferty, L. Bîrzan et al., Astrophys. J. 648, 164 (2006)

L. Metcalfe, D. Fadda, A. Biviano, Space Sci. Rev. 119, 425 (2005)

C.A. Metzler, A.E. Evrard, Astrophys. J. 437, 564 (1994)

S. Muchovej, T. Mroczkowski, J.E. Carlstrom et al., Astrophys. J. 663, 708 (2007)

G. Murante, M. Giovalli, O. Gerhard et al., Mon. Not. R. Astron. Soc. 377, 2 (2007)

J.F. Navarro, C.S. Frenk, S.D.M. White, Mon. Not. R. Astron. Soc. 275, 56 (1995a)

J.F. Navarro, C.S. Frenk, S.D.M. White, Mon. Not. R. Astron. Soc. 275, 720 (1995b)

J. Nevalainen, M. Bonamente, J. Kaastra, Astrophys. J. 656, 733 (2007)

F. Nicastro, S. Mathur, M. Elvis et al., Nature 433, 495 (2005)

B.W. O'Shea, K. Nagamine, V. Springel, L. Hernquist, M.L. Norman, Astrophys. J. Suppl. Ser. 160, 1 (2005)

F. Paerels, J.S. Kaastra, T. Ohashi et al., Space Sci. Rev. (2008). doi:10.1007/s11214-008-9323-6

P.J.E. Peebles, Astron. J. 75, 13 (1970)

V. Petrosian, G. Madejski, K. Luli, Astrophys. J. 652, 948 (2006)

V. Petrosian, A.M. Bykov, Space Sci. Rev. (2008). doi:10.1007/s11214-008-9315-6

V. Petrosian, A.M. Bykov, Y. Rephaeli, Space Sci. Rev. (2008). doi:10.1007/s11214-008-9327-2

M. Plionis, S. Basilakos, Mon. Not. R. Astron. Soc. 329, L47 (2002)

M. Plionis, C. Benoist, S. Maurogordato, C. Ferrari, S. Basilakos, Astrophys. J. 594, 144 (2003)

M. Postman, M. Franx, N.J.G. Cross et al., Astrophys. J. 623, 721 (2005)

W.H. Press, P. Schechter, Astrophys. J. 187, 425 (1974)

M. Ramella, A. Biviano, A. Pisani et al., Astron. Astrophys. 470, 39 (2007)

M. Rauch, J. Miralda-Escudé, W.L.W. Sargent et al., Astrophys. J. 489, 7 (1997)

T.H. Reiprich, H. Böhringer, Astrophys. J. 567, 716 (2002)

Y. Rephaeli, J. Nevalainen, T. Ohashi, A. Bykov, Space Sci. Rev. (2008). doi:10.1007/s11214-008-9314-7

P. Richter, F. Paerels, J.S. Kaastra, Space Sci. Rev. (2008). doi:10.1007/s11214-008-9325-4

K. Rines, A. Mahdavi, M.J. Geller et al., Astrophys. J. 555, 558 (2001)

K. Rines, M.J. Geller, M.J. Kurtz, A. Diaferio, Astron. J. 130, 1482 (2005)

K. Rines, R. Finn, A. Vikhlinin, Astrophys. J. 665, L9 (2007)

P. Rosati, in Clusters of Galaxies: Probes of Cosmological Structure and Galaxy Evolution, ed. by J.S. Mulchaey, A. Dressler, A. Oemler. Carnegie Obs. Astrophys. Ser. (Cambridge Univ. Press, Cambridge, 2004), p. 72 
P. Rosati, S. Borgani, C. Norman, Annu. Rev. Astron. Astrophys. 40, 539 (2002)

M. Rossetti, S. Molendi, Astron. Astrophys. 414, L41 (2004)

B.F. Roukema, P.J. Quinn, B.A. Peterson, B. Rocca-Volmerange, Mon. Not. R. Astron. Soc. 292, 835 (1997)

J.S. Sanders, A.C. Fabian, R.J.H. Dunn, Mon. Not. R. Astron. Soc. 360, 133 (2005)

D.N. Sastry, Publ. Astron. Soc. Pac. 80, 252 (1968)

S. Schindler, A. Diaferio, Space Sci. Rev. (2008). doi:10.1007/s11214-008-9321-8

P. Schneider, in Gravitational Lensing: Strong, Weak and Micro, ed. by G. Meylan, P. Jetzer, P. North. SaasFee Advanced Course, vol. 33 (Springer, Berlin, 2006), p. 1

P. Schuecker, H. Böhringer, T.H. Reiprich, L. Feretti, Astron. Astrophys. 378, 408 (2001)

P. Schuecker, A. Finoguenov, F. Miniati, H. Böhringer, U.G. Briel, Astron. Astrophys. 426, 387 (2004)

H. Shapley, Harvard. Obs. Bull. 838, 3 (1926)

H. Shapley, Proc. Nat. Acad. Sci. Washington 19, 591 (1933)

R.K. Sheth, A. Diaferio, Mon. Not. R. Astron. Soc. 322, 901 (2001)

R.K. Sheth, G. Tormen, Mon. Not. R. Astron. Soc. 308, 119 (1999)

G.P. Smith, T. Treu, R.S. Ellis, S.M. Moran, A. Dressler, Astrophys. J. 620, 78 (2005)

G.F. Smoot, C.L. Bennet, A. Kogut et al., Astrophys. J. 396, L1 (1992)

D.N. Spergel, R. Bean, O. Doré et al., Astrophys. J. Suppl. Ser. 170, 377 (2007)

V. Springel, S.D.M. White, A. Jenkins et al., Nature 435, 629 (2005)

V. Springel, C.S. Frenk, S.D.M. White, Nature 440, 1137 (2006)

R.A. Sunyaev, Ya.B. Zeldovich, Comments Astrophys. Space Phys. 4, 173 (1972)

Y. Takei, J.P. Henry, A. Finoguenov et al., Astrophys. J. 655, 831 (2007)

M. Tegmark, M.R. Blanton, M.A. Strauss et al., Astrophys. J. 606, 702 (2004)

L.S. The, S.D.M. White, Astron. J. 92, 1248 (1986)

P.A. Thomas, H.M.P. Couchman, Mon. Not. R. Astron. Soc. 257, 11 (1992)

R. Valdarnini, Astrophys. J. 567, 741 (2002)

R. Valdarnini, Mon. Not. R. Astron. Soc. 339, 1117 (2003)

G.M. Voit, Rev. Mod. Phys. 77, 207 (2005)

S. von Hoerner, Z. Astrophys. 50, 184 (1960)

N. Werner, J.S. Kaastra, Y. Takei et al., Astron. Astrophys. 468, 849 (2007)

N. Werner, F. Durret, T. Ohashi, S. Schindler, R.P.C. Wiersma, Space Sci. Rev. (2008). doi:10.1007/ s11214-008-9320-9

M.J. West, Mon. Not. R. Astron. Soc. 268, 79 (1994)

S.D.M. White, Mon. Not. R. Astron. Soc. 177, 717 (1976)

M. Wolf, Publ. Astrophys. Inst. Königstuhl-Heidelberg 1, 125 (1902)

D.G. York, J. Adelman, J.E. Anderson Jr. et al., Astron. J. 120, 1579 (2000)

S. Zibetti, S.D.M. White, D.P. Schneider, J. Brinkmann, Mon. Not. R. Astron. Soc. 358, 949 (2005)

F. Zwicky, Helv. Phys. Acta 6, 110 (1933)

F. Zwicky, Astrophys. J. 86, 217 (1937)

F. Zwicky, E. Herzog, P. Wild, M. Karpowicz, C.T. Kowal, Catalogue of Galaxies and Clusters of Galaxies (Pasadena, California Institute of Technology, 1961-1968) 